\title{
EL MERCADO Y LA EDUCACIÓN SUPERIOR
}

\author{
Paulina Dittborn* \\ Fernando Lolas* \\ José Miguel Salazar*
}




\section{RESUMEN}

Luego de examinar las posibles causas de los problemas que enfrenta la educación superior en Chile, este artículo revisa las dos orientaciones que guían a sus instituciones: una perspectiva -más tradicional- que concibe a esta modalidad educacional como institución social productora de bienes públicos y otra -más moderna- que la entiende como una rama industrial, prestadora de servicios y regida por la dinámica del mercado. De estas distintas concepciones surge de manera natural la diferencia entre universidad pública y privada a las que corresponden, consecuentemente, diversos modelos de gestión. La proposición de los autores es recoger elementos de ambas modalidades de instituciones de educación superior y proceder a un modelo que responda a las necesidades nacionales, asegurando la calidad y la fe públicas depositadas en el sistema. Un freno importante a este desafío, sin embargo, está en el marco legislativo que, al respecto, limita a las instituciones tanto en su finalidad como en sus procedimientos de acción.

\section{ABSTRACT}

After examining the possible causes of the problems that higher education faces in Chile, the paper reviews two approaches that guide higher education institutions: a -more traditionalapproach which views higher education as a social institution destined to produce a public good and a-more modern-approach that views it as a line of industry, that renders a service and is governed by market dynamics. From these two approaches the difference governing public and private universities emerges naturally and hence, so do different management models. The author's proposal is to take elements from both institutional models to thus develop a model that addresses national needs, thereby assuring quality and answering to the public faith deposited in the system. An important constraint that limits this challenge, however, lies in the legislative framework which in this respect, inhibits the institutions both in terms of their aims as well as in their procedures for action. 


\section{EL MERCADO Y LA EDUCACIÓN SUPERIOR}

\section{La situación en Chile}

Nuestro sistema permite, por regla general, que las universidades se organicen como entidades estatales autónomas o como corporaciones de derecho privado, recogiendo así la tradición de que la educación superior es un mundo distinto al de las actividades económicas.

Durante los últimos años, ha cundido la percepción de que la educación superior en Chile enfrenta varios problemas y, al mismo tiempo, que un buen sistema de educación superior es una de las mejores herramientas para que el país transite con éxito a integrarse en la llamada "sociedad global del conocimiento". Ello ha puesto de manifiesto la necesidad de poner en marcha distintas medidas para enfrentar tales problemas, proceso al que se han abocado tanto las autoridades como las universidades, y que ha relevado la importancia que la educación superior está cobrando en el debate de las políticas públicas.

Si bien se destaca unánimemente el significativo aumento que ha experimentado la cobertura del sistema luego de las reformas de 1980 y 1990 (que ha pasado de 118.962 alumnos a 521.609, entre 1980 y 2002, conforme a los datos del Ministerio de Educación), existe una extendida preocupación sobre la actualidad, flexibilidad y extensión de los programas académicos de las universidades, el destino de sus egresados en el mercado del trabajo (particularmente en aquellas carreras en que se concentra la mayor cantidad de estudiantes), el desarrollo de la investigación básica y aplicada, los mecanismos de financiamiento de estudios superiores, la efectividad de la gestión 
administrativa y económica de las instituciones, el bajo nivel de protección de los derechos de los estudiantes y la calidad de formación que entregan. Se trata de problemas que, sin embargo, no resultan ajenos a los procesos de masificación de la educación superior, tal como se ha constatado en la experiencia de varios países europeos en décadas anteriores (Maassen et al., 2000).

Sobre las causas de tales problemas en Chile, sin embargo, no existe un consenso muy claro:

a) Algunos consideran que las universidades aún no han sabido advertir y dar una pronta respuesta a los significativos cambios que está experimentando la sociedad chilena, que diseñan y administran sus programas académicos sin considerar oportunamente los requerimientos y demandas del mercado laboral, que no crean conocimiento que agregue verdadero valor a la economía nacional, y que su gestión y planificación económica, financiera y de recursos académicos no son satisfactorias ni responden necesariamente a los objetivos que ellas persiguen.

b) Otros estiman que la sociedad, en su conjunto, no ha asumido responsablemente la expansión de su educación superior, encomendando a las universidades una amplia gama de nuevas tareas y responsabilidades, en distintos ámbitos, sin entregarles los recursos necesarios para que ellas sean cumplidas satisfactoriamente, obligándolas a concentrar una parte significativa de su quehacer en la búsqueda de nuevas alternativas de financiamiento, desdibujando así su misión.

c) Otros, a su vez, consideran que el marco legal vigente está conformado por una sucesión de regulaciones que no resulta armónica ni responde a la actual complejidad de la educación superior, ya que en él no existen adecuadas instancias y mecanismos de coordinación, de financiamiento y de aseguramiento de la calidad que den claridad al conjunto de las universidades (y a la sociedad) acerca de las políticas públicas en marcha y del rol que cada una de ellas puede o debe cumplir en vastas áreas. Esta insuficiencia del marco legal instala una permanente tensión entre la autonomía que se les confiere y las diferentes formas de tutela que sobre ellas se ejerce. 
d) Finalmente, algunos consideran que el Estado no ha sido capaz de poner en práctica mecanismos sistemáticos y estables de asignación de recursos para la educación superior, y de protección de la fe pública involucrada en ella, entregando señales ambiguas y fragmentarias a las instituciones que han limitado su potencial de desarrollo, coartando su iniciativa para adaptarse a la situación presente y proyectar su futuro. Esta percepción también tiende a considerar que el Estado no habría sido capaz de producir una adecuada articulación entre la educación secundaria y la superior, lo que se ha traducido en pobres desempeños de los nuevos estudiantes que acceden a las universidades y ha puesto en duda los niveles de calidad de la formación que imparten las instituciones.

Universidad y sociedad: institución social y actividad económica

Muchas de las causas señaladas han tenido incidencia en el actual estado de cosas, si bien ninguna de ellas lo explica por sí sola. Existe consenso en que una parte importante de los problemas detectados se explican por la transformación que experimenta la educación superior en un doble sentido: el tránsito de una educación superior de elite a una educación superior de masas (Trow, 1974) y una nueva percepción de la importancia que la educación superior tiene para el crecimiento económico de los países (Ginés Mora \& Vila, 2003).

Tal transformación ha sido evaluada desde dos puntos de vista contrapuestos y que surgen de distintas visiones acerca del papel y la relevancia de la educación superior en la vida social. Hay quienes sostienen que esta modalidad educacional es una institución social llamada a producir bienes públicos que, en rigor, no son propiedad de ninguna persona en particular y cuyo acceso y goce no está limitado por consideraciones pecuniarias o de exclusión de grupos. Una burocracia ilustrada, como el ideal de la escuela superior napoleónica, o unos ciudadanos excepcionales, como el sabio que albergaba la universidad humboldtiana, producen, por su sola presencia, un mejoramiento en la calidad de la vida social que no necesariamente depende del talento individual, sino de la institución que lo sustenta, fomenta y cultiva. El derecho al cultivo de los propios talentos, en bien de la sociedad, puede entonces erigirse en principio rector de la fundación de universidades. 
Aún dentro de la heterogeneidad que implica concebir una compleja institución social productora de bienes públicos, esta perspectiva se diferencia de otra que concibe a la institución universitaria básicamente como un sector económico (o una rama industrial) en el campo de los servicios, que genera riqueza y debe desarrollar su quehacer en el contexto de un mercado altamente competitivo (Maassen et al., 2000).

Ambas perspectivas consideran diferentes supuestos sobre las funciones sociales más importantes de la educación superior, los principales problemas que enfrenta y las mejores soluciones o enfoques para abordarlos.

La perspectiva "institución social productora de bienes públicos" considera que las universidades deben alcanzar los objetivos educacionales relacionados con sus actividades esenciales: conservar y proyectar su legado institucional y cumplir importantes funciones relacionadas con toda la sociedad, como la formación de ciudadanos, la preservación del acervo cultural, y la formación del carácter y las habilidades de los estudiantes. Aún dentro de la heterogeneidad de funciones atribuida a las instituciones, se entiende que el lucro o ganancia no es la más esencial y su sostenimiento debiera ser tarea y obligación de todo el cuerpo social.

Por su parte, la posición que considera a la educación superior como una rama de las actividades económicas enseña que las universidades venden bienes y servicios, entrenan a una parte importante de la fuerza de trabajo y fomentan el desarrollo económico de los países. Considera también que la exposición a las fuerzas del mercado, y a otras presiones competitivas, se traducirán en una mejor gestión de las instituciones, una pronta adaptación de los programas de estudio y la máxima flexibilidad y eficiencia en la expectativa de acrecentar la satisfacción del cliente.

La perspectiva de la institución social intenta sustraer a las universidades de las fuerzas de mercado y de la primacía de las demandas económicas de corto plazo en beneficio de responsabilidades sociales más amplias y más trascendentes. A su vez, la perspectiva de la rama productiva está dominada por la preocupación de que la falta 
de habilidad o voluntad para adaptarse a un nuevo contexto se traduzca en irrelevancia de la educación superior y del cultivo del saber para la sociedad global.

La tensión entre estas dos perspectivas ha sido intensa, si bien algo difusa, tanto en el debate internacional como en Chile. Mientras la perspectiva de la institución social puede ser considerada como la representación tradicional de la educación superior, aquella del sector productivo tiende a ser vista como la más moderna. Este último punto de vista incluso ha empezado a ser considerado por el Estado, que ha enfocado su evaluación de las instituciones de educación superior sobre la base de su efectividad y eficiencia.

\section{Una propuesta}

La disyuntiva entre ambas perspectivas puede resultar más aparente que real, sobre todo en relación con el importante rol que ambos puntos de vista tienen que cumplir en el éxito de la transición de la educación superior chilena.

Ello pasa por constatar la gran diversidad que existe al interior del sistema. Hay instituciones estatales, instituciones privadas que tienden a asimilarse a las primeras en varios aspectos, e instituciones privadas creadas con posterioridad a 1980 y que están sujetas a un estatuto general. Suman (hasta el año 2002) 222 entidades entre universidades, institutos profesionales y centros de formación técnica, y en ellas participa activamente la gran mayoría de grupos e instituciones sociales más representativos y prestigiosos, y nuestros más respetados y preparados ciudadanos. Los recursos que administran provienen del Estado (que destinó, por distintas vías, 285.744 millones de pesos a la educación superior durante 2003), pero también de los estudiantes y sus familias, y de las más diversas entidades y organizaciones que apoyan a las instituciones por motivos muy variados.

Tal variedad en la propiedad, participación activa, alumnado y financiamiento de las instituciones funda una de las mayores fortalezas que presenta actualmente la educación superior: su diversidad. Al mismo tiempo, ello da cuenta de que la idea general de universidad 
hace hoy difícil comparar instituciones muy diversas, tanto en objetivos y propósitos como en trayectoria, recursos humanos y materiales con que cuentan, funciones que desempeñan, actividades que realizan y áreas del conocimiento que abarcan, entre otros aspectos.

La diversidad es tan amplia entre las universidades (eran más de sesenta al 2003), que ha sido necesario recurrir a diversos criterios para identificar grupos de instituciones que presentan características afines. Así, distintos análisis y publicaciones ${ }^{1}$ hablan de instituciones complejas e instituciones puramente docentes, o de entidades de investigación, docentes extensivas, docentes comprensivas, docentes concentradas o especializadas.

Con todo, quizás el aspecto que permite diferenciar más nítidamente a las universidades es la forma en que ellas se gestionan y los objetivos que efectivamente persiguen.

Una clasificación fundada en este criterio podría dar cuenta de la existencia de una distancia entre los hechos y el marco regulatorio a que las universidades están sujetas, considerando que la ley define a la universidad como "una institución de educación superior, de investigación, raciocinio y cultura que, en el cumplimiento de sus funciones, debe atender adecuadamente los intereses y necesidades del país, al más alto nivel de excelencia" y que, por ello, todas deberían perseguir en teoría los mismos objetivos, organizándose acorde a una gama de modelos de gestión definidos en función de la cultura interna de cada institución. Empero, ello debe entenderse más como una interrogante abierta sobre la pertinencia del actual marco legal, que como una crítica a las instituciones y la forma en que ellas se conducen.

Existe una evidente relación entre los objetivos que las universidades persiguen y los modelos de gestión que ellas asumen (entendidos éstos como la forma en que las instituciones se conducen internamente y se relacionan con la sociedad, en términos de los programas académicos y de capacitación que ofrecen, las acciones de extensión que llevan a cabo y las distintas actividades de investigación que puedan realizar). Tal relación está determinada por la forma más

$\overline{1}$ Ejemplo de ello es el ranking publicado en el 2003 por la revista Qué Pasa. 
efectiva de dar consecución a los objetivos de cada universidad, de manera que sus modelos de gestión tienden a ser definidos (explícita o implícitamente) en función de los propósitos reales que cada institución tenga, siendo unos modelos de gestión más aptos que otros para el logro de los mismos.

En ese contexto, es posible advertir dos tipos básicos de finalidades mediatas que las instituciones persiguen, cuyas causas más remotas pueden ser muy heterogéneas. A su vez, existen dos grandes modelos de gestión universitaria, si bien hay, entre ellos, varias soluciones intermedias.

Existen universidades (preferentemente, aunque no exclusivamente) públicas cuya finalidad prioritaria apunta a constituir un espacio de formación, debate e investigación del más alto nivel para el cumplimiento de los intereses y necesidades nacionales, o para la creación, preservación, cultivo, transmisión y búsqueda del conocimiento, priorizando el desarrollo de la conciencia crítica en un ámbito pluralista, en el que imperen la libertad de expresión y la tolerancia. Para ello adoptan estructuras de gobierno que se articulan sobre la base de organizaciones cooperativistas (apoyadas, sin embargo, en una amplia y férrea defensa de la autonomía universitaria enfrente del Estado), en las que las comunidades de docentes e investigadores cuentan con un amplio poder real al designar directamente a una gran parte de las autoridades superiores. Al mismo tiempo, estas instituciones tienden a tener una clara conciencia del rol que cumplen en la sociedad, en el sentido de ser un importante espacio de lo público, en el que los valores de la diversidad y el ejercicio de los derechos y deberes inherentes a la ciudadanía son vivamente encarnados, promoviendo, al mismo tiempo, la igualdad de oportunidades y apoyando decididamente el desarrollo de la ciencia y la tecnología. Por lo mismo, las demandas del mercado laboral y la gestión de mercado tienen un bajo nivel de incidencia en su quehacer, sujeto a sistemas de control estatal bastante estrictos.

Por su parte, existen instituciones (prioritariamente privadas) cuya finalidad principal es preparar y actualizar la fuerza laboral en todos sus niveles, profundizando y fortaleciendo la estructura económica del país, esperando recibir por ello una legítima ganancia. 
Tales universidades tienen un claro modelo de gestión de mercado (ya sea service university, entrepreneurial university, innovative university, virtual university o network university, u otros esquemas flexibles), con un estilo de administración que apunta a distanciar la gestión de los académicos, radicándola en autoridades superiores dotadas de un nítido liderazgo y que surgen externamente a la organización académica, A su vez, se orientan a priorizar la satisfacción de sus distintos "clientes" y a definir flexiblemente su estructura organizacional (y los servicios que ofrecen) sobre la base de la interacción de mercado (esto es, el comportamiento de las otras instituciones similares y las tendencias de matrícula observadas en los postulantes a la educación superior). Se trata de una lógica de gestión de mercado que ya es una realidad en la educación superior chilena y que también se aplica en otros países hace algún tiempo, ejemplo de lo cual son las for-profit universities en Estados Unidos (si bien éstas están sujetas a un estatuto especial en dicho país).

De esa forma, y según el carácter que se confiera a la investigación (básica o aplicada, de apoyo para la docencia o para la creación de nuevo conocimiento) y a la extensión (abierta a la comunidad o vinculada al financiamiento), sólo algunas instituciones se encuentran en posición de llevar a cabo actividades en ésta o aquélla, en función de las finalidades que estén dispuestas a cumplir y el modelo de gestión que utilicen. A su vez, aquellas demandas nuevas que se plantean a la educación superior, como las relativas a la educación continua y a distancia, podrían ser mejor atendidas en universidades públicas que en aquellas instituciones que expresamente se orientan a la satisfacción de las demandas del mercado del trabajo.

Se trata, sin duda, de dos sistemas complementarios de gestión de universidades (que se traslapan en varios niveles, particularmente en la docencia), a través de los cuales la educación superior proyecta su quehacer en el tiempo y adquiere mayor centralidad en el desarrollo del país, en la medida en que propende a hacerse cargo de las demandas de los distintos sectores económicos, junto con asumir los requerimientos más amplios que la sociedad civil le plantea. De esa forma, y por medio de la autorregulación, cada institución tiende a hacerse cargo de una parte de las expectativas que se tienen del sistema completo, permitiendo a éste cumplir todos los roles que la sociedad confía a la educación superior. 
Esta visión integrada del mundo universitario, que considera distintos tipos de instituciones con diversos sistemas de gestión y que cumplen distintas finalidades, articula un sistema de educación superior plural y vigoroso, apto para enfrentar los desafíos que la masificación y los nuevos modelos de desarrollo le exigen. A su vez, le permiten encarar las nuevas demandas que se plantearán en el futuro y que dicen relación con una mayor expansión de la matrícula, una profundización de la diversificación de la formación que ofrecen (tanto en contenido como en estrategias pedagógicas), la globalización de la educación superior, los cambios que se introducirán en los sistemas de financiamiento y de gobierno de las universidades, y la profesionalización de la gestión universitaria (Teichler, 2003).

En virtud de lo anterior, el desafío que una visión integrada del sistema universitario enfrenta consiste en mejorar crecientemente la calidad de los procesos y desempeños, disminuyendo al mismo tiempo los riesgos que cada punto de vista presenta por separado, por medio de una adecuada coordinación del sistema de educación superior que integre a todos sus partícipes al más alto nivel y, sobre todo, reconozca la posibilidad legítima de distintos propósitos y modelos de gestión a su interior.

Para ello, debe tenerse presente que, en Chile, la legislación impone a las universidades el cumplimiento de un grupo de finalidades predefinidas que no resulta idóneo si se busca proteger y fomentar esta diversidad. En ese marco legal, las universidades sólo son instituciones sin fines de lucro que desarrollan docencia, investigación y extensión al más alto nivel. Tal definición tiende a limitar formalmente la pluralidad de objetivos y modelos de gestión entre las universidades, poniendo en entredicho su diversidad y la expansión de la educación superior.

En consecuencia, será de central importancia revisar el actual marco regulatorio de la educación superior a fin de reconocer las distintas realidades que en ésta se plantean, permitiendo que las instituciones que así lo estimen puedan formalizar debidamente objetivos y modelos de gestión orientados al mercado, incluida la posibilidad de que sus propietarios puedan obtener utilidades por su quehacer. 
Al mismo tiempo, y en concordancia con lo anterior, será necesario fortalecer los mecanismos para resguardar la fe pública depositada en el sistema de educación superior y asegurar su calidad en un contexto de amplia diversidad, definiendo instrumentos efectivos para proteger a la comunidad de eventuales fraudes que se puedan incubar en el sistema universitario, y privilegiar los medios más efectivos para la articulación de las instituciones que forman parte de él. Finalmente, es esencial que el nuevo marco legislativo permita el financiamiento de los estudios superiores en todos los niveles del sistema, para permitir a los estudiantes que cursen estudios en las instituciones que ellos elijan, en un contexto de amplia diversidad, dado que ello será decisivo para la expansión y proyección de la educación superior.

En la medida en que el sistema de educación superior asuma tales desafíos, quedará en un mejor pie para superar exitosamente los problemas que hoy le aquejan. 


\section{REFERENCIAS BiBLIOGRÁFICAS}

Ginés Mora, J. \& Vila, L., "The economics of Higher Education", in Begg, Roddy, ed., The Dialogue Between Higher Education Research and Practice, Dordrecht, Netherlands, Kluwer, 2003, pp. 121-131.

Maanssen, P., Fulton, O., Hölttä, S., Amaral, A., Boffo S., Verhoeven, J., Pechar, H. \& Kyvik, S., Governmental Policies and Programmes for Strengthening the Relationship Between Higher Education Institutions and the National Economy (HEINE Report), TSER Programme, 2000.

Teichler, U., "The future of Higher Education and the future of Higher Education Research", Tertiary Education and Management, September 2003, vol. 9, iss. 3, pp. 171-185(15).

Trow, M., "Problems in the transition from elite to mass Higher Education", in Policies for Higher Education, General Report to the Conference on the Future Structure of Post-Secondary Education, Paris, OECD, 1971, pp. 51-101. 